\title{
Attempt to Describe Phase Slips by Means of an Adiabatic Approximation
}

Jorge Berger ( $\sim$ jorge.berger@braude.ac.il )

Ort-Braude College

\section{Research Article}

Keywords: Phase Slips, Adiabatic Approximation, plausibility test

Posted Date: July 29th, 2021

DOl: https://doi.org/10.21203/rs.3.rs-753971/v1

License: (c) (i) This work is licensed under a Creative Commons Attribution 4.0 International License. Read Full License 


\title{
Attempt to describe phase slips by means of an adiabatic approximation
}

\author{
Jorge Berger ${ }^{1, *}$ \\ ${ }^{1}$ Ort-Braude College, Physics and Optical Engineering, Karmiel, 2161002, Israel \\ *jorge.berger@braude.ac.il
}

\begin{abstract}
As a plausibility test for the feasibility of extension of the quasiclassical Keldysh-Usadel technique to slowly varying situations, we assess the influence of the time-derivative term in the time-dependent Ginzburg-Landau equation. We consider cases in which the superconducting state in a nanowire varies slowly, either because the voltage applied on it is small, or because most of phase drift takes place next to the boundaries. An approximation without this time derivative can describe the superconducting state away from phase slips, but is unable to predict the value or the existence of a critical voltage at which evolution becomes non-stationary.
\end{abstract}

\section{Introduction}

Superconducting nanocircuits and nanodevices have been available for decades. ${ }^{1-6}$ Here we will focus on their most basic component: a nanowire. When a voltage is applied between the ends of a superconducting wire, a phase difference between them builds up. Eventually, a phase slip (or a set of phase slips) occurs, i.e., superconductivity vanishes somewhere in the wire, enabling discontinuities in the phase by some multiple of $2 \pi .^{7}$ In this study we do not consider phase slips that are due to thermal $^{8}$ or quantum ${ }^{9,10}$ fluctuations.

The system we consider consists of a superconducting wire that connects two conducting banks. The wire is thin compared to the coherence length and the banks are at equilibrium and kept at fixed potentials. Theoretical analysis of this system requires a theory that takes time dependence into account. The available theory is the the Kramer--Watts-Tobin model, ${ }^{11-13}$ which is valid only close to the critical temperature $T_{c}$. Among the numerous studies with validity close to $T_{c}$, some consider infinite wires, ${ }^{14}$ some consider finite wires carrying a fixed current, ${ }^{15,16}$ and some consider finite wires that withstand a fixed voltage. ${ }^{17}$ It should be noticed that the voltage-current characteristic of a superconducting wire in the case that the current is kept constant differs qualitatively from the case in which the voltage is kept constant. ${ }^{18,19}$

By following in time the state of the wire close to $T_{c}$, an established feature is that it converges either to a stationary regime, in which all measurable quantities become independent of time, or to an oscillatory regime, in which phase slips occur. In many situations, the final regime depends on parameters such as the temperature, the length, or the driven current, and the research objective is to find the regime diagram.

On the other hand, the most interesting temperature range for application purposes is far below $T_{c}$. In this range, the available theory is the quasiclassical Keldysh-Usadel technique (QKU), ${ }^{20,21}$ that relies on a one-time Green's function formalism and is valid only for stationary situations; time dependence would involve two-time Green's functions, that would be too cumbersome to deal with. As a consequence, studies in this range ${ }^{22-24}$ have to assume a priori that the system is in a stationary regime.

The idea studied in the present study is: Could we extend the QKU technique to non stationary situations by means of an adiabatic approximation $?^{25,26}$ Namely, are there situations in which the state of the system evolves sufficiently slowly to enable a reasonable description by means of a theory that ignores time dependence? We will test this idea invoking the available time-dependent theory. Since the behavior of a superconducting wire is similar whether slightly below or far below $T_{c}$, the success or failure of the adiabatic approximation (AA) in one of these ranges provides an educated guess for the expected suitability of this approximation in the other range.

We will consider two candidates for slow evolution: (i) a case of small applied voltage, so that the Josephson frequency is small, and (ii) a case of continuous passage between a stationary and an oscillatory regime; by taking parameters that approach the stationary regime, the oscillation period diverges. 


\section{Mathematical model}

For a one-dimensional system, the vector potential can be taken as zero. Then the evolution equation becomes ${ }^{11}$

$$
\frac{u}{\left[1+\gamma^{2}|\psi|^{2}\right]^{1 / 2}}\left[\frac{\partial}{\partial t}+i \mu+\frac{\gamma^{2}}{2} \frac{\partial|\psi|^{2}}{\partial t}\right] \psi=\frac{\partial^{2} \psi}{\partial x^{2}}+\left(1-|\psi|^{2}\right) \psi .
$$

Here $\psi$ is the order parameter, that determines the supercurrent density, $u$ and $\gamma$ are material parameters, $x$ is the position in units of the coherence length $\xi, t$ is the time in units of $\xi^{2} / D u$, where $D$ is the diffusion coefficient, and $\mu$ is the electrochemical potential in units of $\hbar D u / 2 e \xi^{2}$. The current density $j$, in units of $\hbar \sigma D u / 2 e \xi^{3}$, where $\sigma$ is the normal conductivity, is given by

$$
j=\operatorname{Im}\left[\psi^{*} \partial \psi / \partial x\right]-\partial \mu / \partial x
$$

and invoking electroneutrality,

$$
\partial^{2} \mu / \partial x^{2}=\partial \operatorname{Im}\left[\psi^{*} \partial \psi / \partial x\right] / \partial x
$$

Since we are not interested in the accuracy of the model, but rather in how close the AA reproduces it, and since varying $\gamma$ does not lead ${ }^{16}$ to qualitative changes in the regime diagram, we set $\gamma=0$ and Eq. (1) becomes the time-dependent GinzburgLandau equation. Moreover, from the discussion in Ref. 17 we can conclude that the influence of $u$ is also weak and take $u=1$. Thus, Eq. (1) reduces to

$$
\left[\frac{\partial}{\partial t}+i \mu\right] \psi=\frac{\partial^{2} \psi}{\partial x^{2}}+\left(1-|\psi|^{2}\right) \psi
$$

This is the evolution equation used in Ref. 17. Equations (3) and (4) have to be complemented with boundary conditions. For a wire of length $2 L$ with applied voltage $V$, we write $-L \leq x \leq L$ and take the potential equal to $\pm V / 2$ at the ends. Consistently, the order parameter at the banks has to be $r \exp (\mp i V t / 2)$, where $r$ represents the superconductivity strength of the banks; in particular, if the banks are made of the same material as the wire, then $r=1$, and if they are normal metals, then $r=0$. In summary, we take the boundary conditions

$$
\mu(t, \pm L)=\mp V / 2, \quad \psi(t, \pm L)=r e^{ \pm i V t / 2} .
$$

Equations (3)-(5) will constitute our time-dependent model and the AA will be obtained by just dropping the timederivative term in Eq. (4). When comparing solutions of these equations, it has to be borne in mind that the transformation $\{t \rightarrow t+2 \pi / V, \psi \rightarrow-\psi\}$ leads to an equivalent solution. In the following examples, we will take $L=4$.

\section{Superconducting banks}

This situation is of limited interest, since we know in advance that phase slips must occur in order to avoid divergence of the gradient of the phase in the wire. The main value of this section is that it will help us understand and appreciate the following section.

We take $r=1$ in the boundary conditions (5) and, in order to have a slowly varying state, we take a small value of $V$. In our units, "small" means $V \ll 1$, and we thus take $V=0.01$.

The results that we present for the time-dependent model correspond to times after convergence to a clearly defined regime. Equations (3)-(5) support the symmetry $\psi(t,-x)=\psi^{*}(t, x), \mu(t,-x)=-\mu(t, x)$, and the results we obtained do not break this symmetry, so that it suffices to present our results for $x \geq 0$.

Figure 1a is a contour plot of $|\psi(t, x)|$ (according to the time-dependent model). As expected from Ref. 17, there are phase slips at the center of the wire, that occur periodically with a period $2 \pi / V$. Figure $1 \mathrm{~b}$ compares $\psi(t, 0)$ according to the time-dependent model (continuous line) with $\psi(t, 0)$ according to the AA (dashed line). Note that $\psi(t, 0)$ is real. Since at the boundaries $x= \pm L$ there is no difference between the model and the approximation, the agreement is expected to be better for $x \neq 0$. We see that there are time ranges where the AA yields three solutions, so that in order to compare with the model we require some interpretation. The natural interpretation regards the middle branch as a set of unstable solutions, whereas solutions in the upmost and the lowermost branches can be stable. At times for which an end of a stable branch is reached, the state of the system has to jump to the other branch, as shown by the purple arrows. We see that the approximated and the model solutions are practically indistinguishable, except close to the phase slips, where the decay of the model solution is gradual. We may now inquire for the origin of the discrepancy introduced when we drop $\partial \psi / \partial t$, which is expected to be small for $V \ll 1$. The answer is provided by Fig. 1c: although $\partial \psi / \partial t$ is small most of the time, it is not small close to the phase slips.

The time-average of the current density is roughly $10 \%$ smaller in the case of the AA than for the time-dependent model. 

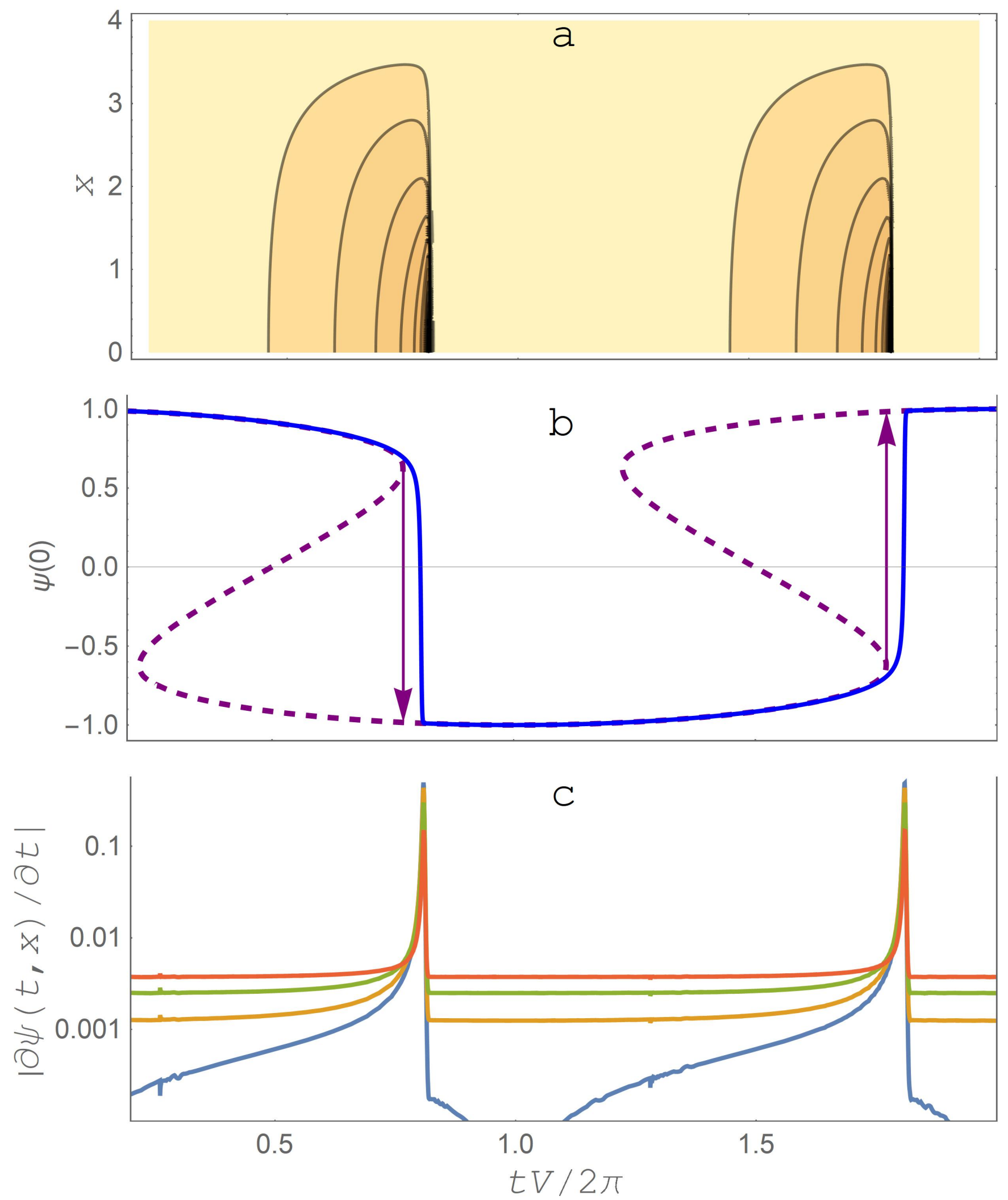

Figure 1. Case $r=1, V=0.01$. The abscissa is common to all panels. a Contour plot of $|\psi(t, x)|$. Darker colors stand for smaller $|\psi|$. $\mathbf{b} \psi(t, 0)$ according to the time-dependent model (continuous, blue) and to the adiabatic approximation (dashed, purple). The arrows indicate discontinuous passage to another branch. $\mathbf{c}|\partial \psi / \partial t|$ as a function of $t$. Blue: $x=0$; orange: $x=1$; green: $x=2$; red: $x=3$. 

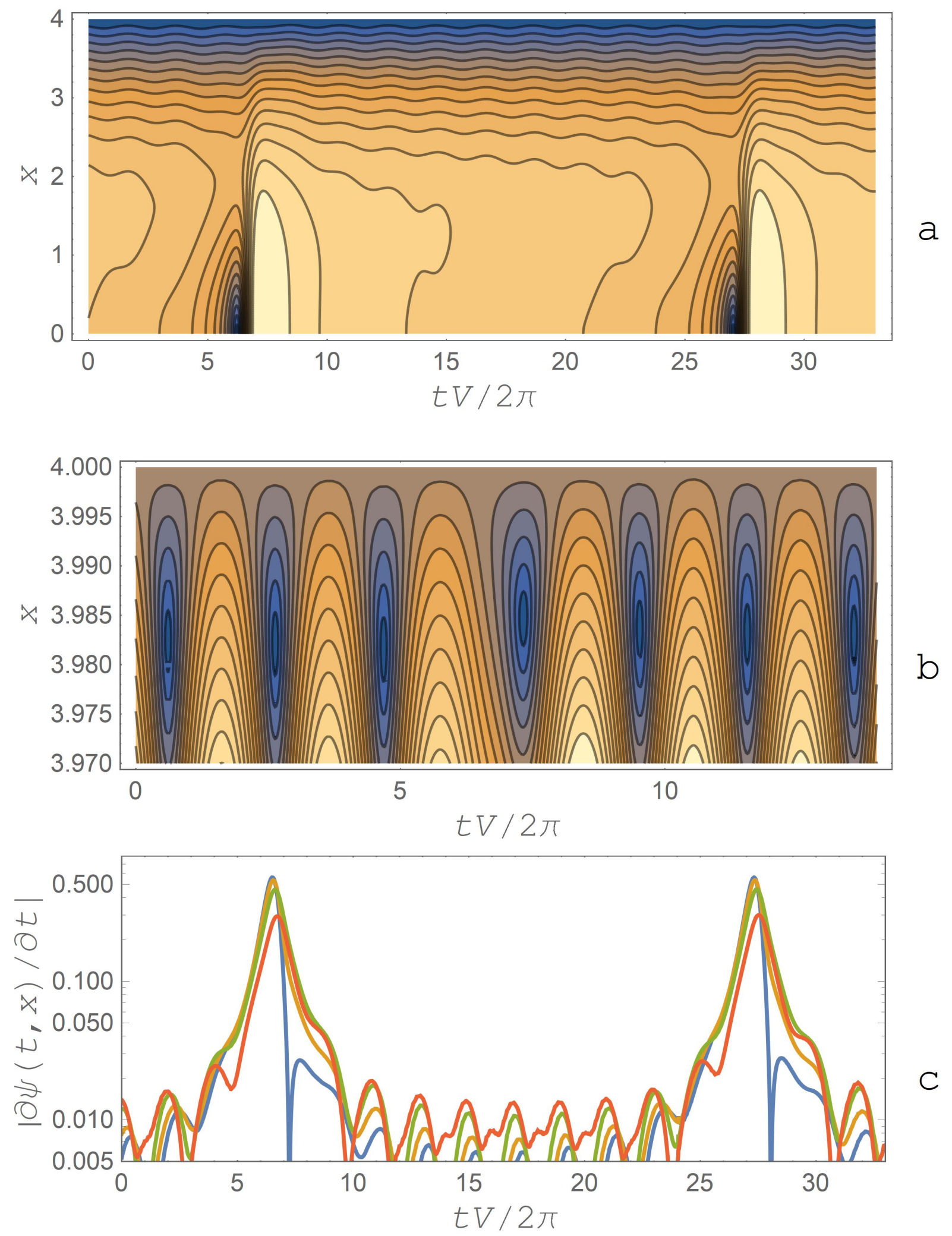

Figure 2. Case $r=0.01, V=2.22$. a Contour plot of $|\psi(t, x)|$ that contains two significant phase slips at $x=0$. b Examples of irrelevant phase slips, that quasi-periodically occur at a distance of $\sim 0.02$ from each of the boundaries, with a repetition time of $\sim 4 \pi / V$. The maximum value of $|\psi|$ in this panel is about 30 times smaller than the maximum in panel $\mathbf{a}$. Panel $\mathbf{b}$ is actually part of panel $\mathbf{a}$, but is not visible in the latter. $\mathbf{c}|\partial \psi / \partial t|$, with the same color meanings as in Fig. 1c. 


\section{Quasi-normal banks}

A normal metal bank is usually analyzed by taking $r=0$. However, in this case the phase at the boundaries becomes undefined, and we have no way to assess the influence of the Josephson evolution of the phase. In order to have well defined phases, we will consider instead quasi-normal banks, such that $0<r \ll 1$. Quasi-normal boundary conditions are not less realistic than strictly normal boundary conditions, since the assumption that the points at $x= \pm L$ are in equilibrium despite the fact the current density does not vanish at them is an idealization anyway. In the following, we take $r=0.01$.

When dealing with quasi-normal banks, we have to distinguish between significant phase slips, that take place where $|\psi(t, x)|$ is usually large, and irrelevant phase slips, at which $\psi(x, t)=0$ for values of $x$ such that the order parameter is anyway small at all times. Examples of significant phase slips are shown in Fig. 2a and examples of irrelevant phase slips are shown in Fig. 2b.

By solving numerically Eqs. (3)-(5) in the range $0 \leq V \leq 3.55$, we find that there is a critical voltage $V_{c} \approx 2.195$ such that for $V<V_{c}$ there are no significant phase slips, whereas for $V>V_{c}$ a phase slip occurs at $x=0$, with repetition time

$$
T \approx \beta / \sqrt{V-V_{c}},
$$

where $\beta=9.22$. It follows that the most promising region for success of the AA lies above and close to $V_{c}$.

Range $V<V_{c}$

We performed calculations for $V=2.15$, for a long period of time. At the boundaries, $|\partial \psi / \partial t|=r V / 2$, and we found that $|\partial \psi / \partial t|$ decreases as $|x|$ decreases. Accordingly, $\partial \psi / \partial t$ is small everywhere, and we can expect that the AA should lead to accurate results.

We found that, for fixed $x, \psi(t, x)$ is a function of time that slightly oscillates around an average value. The average value coincides within 0.01 units for the model and for the AA, but the amplitude of the oscillations can be up to ten times larger in the case of the approximation.

\section{Range $V>V_{c}$}

Figure 2 presents some of our results for $V=2.22$, just slightly above $V_{c}$. The repetition time is $\sim 59$, naively suggesting a very slow variation. Unfortunately, Fig. 2c shows that $\partial \psi / \partial t$ is not small during a large fraction of the time, much larger than in the case of Fig. 1c.

Before we go on, let us spell out what we could expect. Let us assume that significant phase slips occur only at $x=0$. In the AA, time enters the problem only through Eq. (5), which just covers a time period of extent $4 \pi / V$. If the time lapse between consecutive phase slips is $4 n \pi / V$, where $n$ is a natural number, then there must be at least $n$ branches of stable solutions with $\psi(t, 0)>0$, such that the passage between branches is determined by continuity, and $n$ branches with $\psi(t, 0)<0$, so that there are at least $2 n$ stable branches. For a time lapse in the range between $4(n-1) \pi / V$ and $4 n \pi / V$, there should be at least $2 n$ branches, where one of them becomes unstable before filling the entire range allowed by the periodicity of the boundary conditions. In a polar graph where $|\psi(t, 0)|$ is the radius and $V t / 2$ is the angle, a phase slip would be described as part of an orbit that spirals into the origin, possibly backwards in time.

For $V=2.22$, we found three branches of solutions for the AA. For one of them $\psi(t, 0) \sim 0.7$, there is a twin branch for $\psi(t, 0)<0$, and there is a branch such that the wire is quasi-normal everywhere $\left[\psi(t, x)\right.$ is of the order of $\left.10^{-2}\right]$ and irrelevant phase slips occur with period $2 \pi / V$. We looked for solutions such that $0.05 \leq|\psi(t, 0)| \leq 0.65$ for some $t$, and none was found. Within numeric accuracy, the orbits for $|\psi(t, 0)| \sim 0.7$ are closed. These results indicate that if there exists a critical voltage $V_{c}$ in the AA, then $V_{c}>2.22$.

\section{Time between phase slips in the adiabatic approximation}

We tried to evaluate the time elapsed between consecutive significant phase slips using the AA, for applied voltages considerably larger than 2.22, but were unable to find any quasi-periodic regime similar to the one described in Fig. 2. As will become clear below, this approach was doomed to failure.

To overcome this difficulty, we considered "gradual adiabatization," by multiplying $\partial \psi / \partial t$ in Eq. (4) by a factor $\alpha$. By doing this, the AA is obtained in the limit $\alpha \rightarrow 0$. In Eq. (6), $V_{c}$ and $\beta$ will now be functions of $\alpha$.

Figure 3 shows that the time between significant phase slips can be fitted by taking $V_{c}$ independent of $\alpha$ and $\beta$ proportional to $\alpha$. This result suggests that periodic significant phase slips are not expected in the AA, since, if they were present, the time elapsed between them would have to vanish (whatever that means).

\section{Discussion}

Under the assumption that the Kramer-Watts-Tobin model provides a qualitative representation of how a superconducting state evolves in a nanowire, an adiabatic approximation can fairly describe the state of a nanowire for low applied voltages and away 


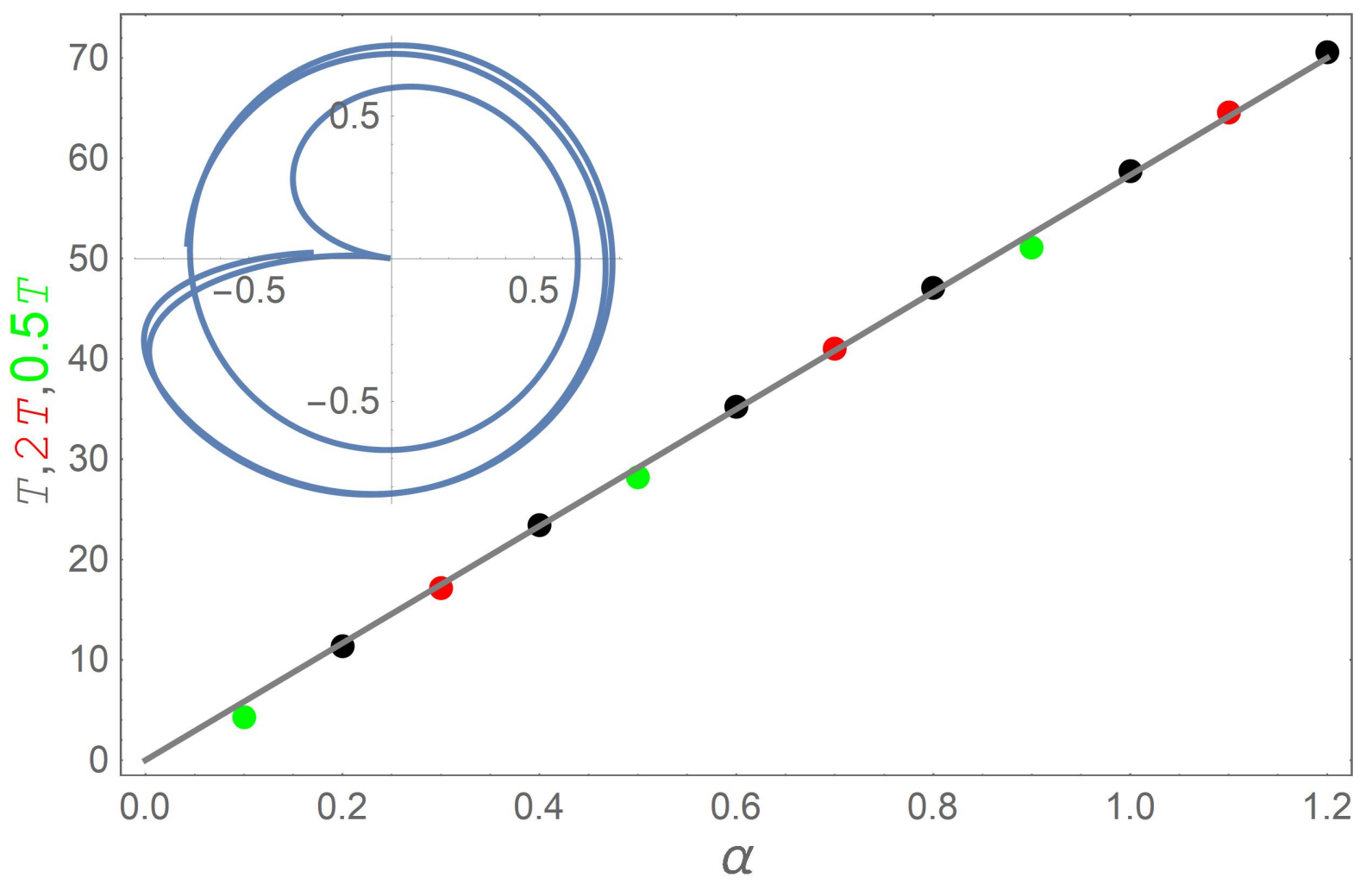

Figure 3. Time between consecutive significant phase slips when the time derivative in Eq. (4) is multiplied by the factor $\alpha$. In all cases, $V_{c}=2.195$. Black: $V=V_{c}+0.025$. Red: $V=V_{c}+4 \times 0.025$; following Eq. (6), $2 T$ is plotted in order to enter a common line. Green: $V=V_{c}+0.025 / 4, T / 2$ is plotted. The straight line describes $58.33 \alpha$. Inset: polar graph with radius $|\psi(t, 0)|$ and angle $V t / 2$ for $V=2.22$ and $\alpha=0.2$; the orbit covers a lapse of time $12 \pi / V \approx 1.5 T$. 
from the moments at which phase slips occur. This approximation could also describe the superconducting state in the case of quasi-normal banks, as long as there are no phase slips far from the banks. However, the approximation fails during the phase slips and, most disappointingly, it is unable to predict whether there is a critical voltage at which the evolution of the superconducting state switches from a quasi-stationary to a quasi-periodic regime.

\section{Numerical details}

All the differential equations were solved using the built-in function NDSolve in Mathematica, and our strategies were adapted for its use.

In the case of the time-dependent model, we took advantage of the "method of lines." For this purpose, we added a small term $\delta \partial \mu / \partial t$, with $0<\delta \ll 1$, at the right hand side of Eq. (3), and verified that our results were practically independent of $\delta$ and that Eq. (3) was actually obeyed.

In the case of the AA, we found that minimization was more stable than direct use of NDSolve with Dirichlet boundary conditions. For stable branches and fixed $t$, we minimized $F[\psi(0), \partial \psi / \partial x(0), \partial \mu / \partial x(0) ; t]=\left|\psi(L)-r e^{i V t / 2}\right|^{2} / r+\mid \mu(L)+$ $V /\left.2\right|^{2} ; \psi(L)$ and $\mu(L)$ were evaluated as functions of the values at $x=0$, using NDSolve. Along and near the unstable branches, we fixed $\psi(0)$ rather than $t$ and minimized $H[\partial \psi / \partial x(0), \partial \mu / \partial x(0) ; \psi(0)]=[|\psi(L)|-r]^{2} / r+|\mu(L)+V / 2|^{2} ; t$ was then obtained from the phase of $\psi(L)$.

\section{References}

1. Foley, C.P. \& Hilgenkamp, H. Why NanoSQUIDs are important: an introduction to the focus issue. Supercond. Sci. Technol. 22, 064001 (2009). https://doi.org/10.1088/0953-2048/22/6/064001

2. Wernsdorfer, W. From micro- to nano-SQUIDs: applications to nanomagnetism. Supercond. Sci. Technol. 22, 064013 (2009). https://doi.org/10.1088/0953-2048/22/6/064013

3. You, J. \& Nori, F. Atomic physics and quantum optics using superconducting circuits. Nature 474, 589-597 (2011). https://doi.org/10.1038/nature10122

4. Granata, C. \& Vettoliere, A. Nano Superconducting Quantum Interference device: a powerful tool for nanoscale investigations. Phys. Rep. 614, 1-69 (2016). https://doi.org/10.1016/j.physrep.2015.12.001

5. Levenson-Falk, E. M., Antler N. \& Siddiqi, I. Dispersive nanoSQUID magnetometry. Supercond. Sci. Technol. 29, 113003 (2016). https://doi.org/10.1088/0953-2048/29/11/113003

6. Martínez-Pérez, M. J. \& Koelle, D. NanoSQUIDs: Basics \& recent advances. Physical Sciences Reviews 2, 20175001 (2017). https://doi.org/10.1515/psr-2017-5001

7. Langer, J. S. \& Ambegaokar, V. Intrinsic resistive transition in narrow superconducting channels. Phys. Rev. 164, 498-510 (1967). https://doi.org/10.1103/PhysRev.164.498

8. McCumber, D. E. \& Halperin, B. I. Time scale of intrinsic resistive fluctuations in thin superconducting wires. Phys. Rev. B 1, 1054-1070 (1970). https://doi.org/10.1103/PhysRevB.1.1054

9. Lau, C. N., Markovic, N., Bockrath, M., Bezryadin, A. \& Tinkham, M. Quantum phase slips in superconducting nanowires. Phys. Rev. Lett. 87, 217003 (2001). https://doi.org/10.1103/PhysRevLett.87.217003

10. Arutyunov, K.Yu., Golubev, D. S. \& Zaikin, A. D. Superconductivity in one dimension. Physics Reports 464 1-70 (2008). https://doi.org/10.1016/j.physrep.2008.04.009

11. Kramer, L. \& Watts-Tobin, R. J. Theory of dissipative current-carrying states in superconducting filaments. Phys. Rev. Lett. 40, 1041-1044 (1978). https://doi.org/10.1103/PhysRevLett.40.1041

12. Watts-Tobin, R.J., Krähenbühl, Y. \& Kramer, L. Nonequilibrium theory of dirty, current-carrying superconductors: phaseslip oscillators in narrow filaments near $T_{c}$. J. Low Temp. Phys. 42, 459-501 (1981). https://doi.org/10.1007/BF00117427

13. Ivlev, B. I. \& Kopnin, N. B. Electric currents and resistive states in thin superconductors, Adv. Phys. 33, 47-114 (1984). https://doi.org/10.1080/00018738400101641

14. Kramer, L. \& Rangel, R. Structure and properties of the dissipative phase-slip state in narrow superconducting filaments with and without inhomogeneities. J. Low Temp. Phys. 57, 391-414 (1984). https://doi.org/10.1007/BF00681200

15. Rubinstein, J., Sternberg, P. \& Ma, Q. Bifurcation diagram and pattern formation of phase slip centers in superconducting wires driven with electric currents. Phys. Rev. Lett. 99, 167003 (2007). https://doi.org/10.1103/PhysRevLett.99.167003

16. Berger, J. Influence of the boundary conditions on the current flow pattern along a superconducting wire. Phys. Rev. B 92, 064513 (2015). https://doi.org/10.1103/PhysRevB.92.064513 
17. Kim, J., Rubinstein, J., \& Sternberg, P. Length/voltage phase diagram for a thin superconducting wire subjected to an applied voltage. Physica C 470, 630-634 (2010). https://doi.org/10.1016/j.physc.2010.06.004

18. Vodolazov, D.Y., Peeters, F.M., Piraux, L., Mátéfi-Tempfli, S. \& Michotte, S. Current-voltage characteristics of quasione-dimensional superconductors: an S-shaped curve in the constant voltage regime. Phys. Rev. Lett. 91, 157001 (2003). https://doi.org/10.1103/PhysRevLett.91.157001

19. Michotte, S., Mátéfi-Tempfli, S., Piraux, L., Vodolazov, D. Y. \& Peeters, F. M. Condition for the occurrence of phase slip centers in superconducting nanowires under applied current or voltage. Phys. Rev. B 69, 094512 (2004). https://doi.org/10.1103/PhysRevB.69.094512

20. Belzig, W., Wilhelm, F. K., Bruder, C., Schön, G. \& Zaikin, A. D. Quasiclassical Green's function approach to mesoscopic superconductivity. Superlatt. Microstruct. 25, 1251-1288 (1999). https://doi.org/10.1006/spmi.1999.0710. Corrigendum: Superlatt. Microstruct. 35, 157 (2004) https://doi.org/10.1016/j.spmi.2004.01.003

21. Kamenev, A. \& Levchenko, A. Keldysh technique and non-linear $\sigma$-model: basic principles and applications, Adv. Phys. 58, 197-319 (2009). http://dx.doi.org/10.1080/00018730902850504

22. Boogaard, G. R., Verbruggen, A. H., Belzig, W. \& Klapwijk, T. M. Resistance of superconducting nanowires connected to normal-metal leads. Phys. Rev. B 69, 220503 (2004). https://doi.org/10.1103/PhysRevB.69.220503

23. Keizer, R. S., Flokstra, M. G., Aarts, J. \& Klapwijk, T. M. Critical voltage of a mesoscopic superconductor. Phys. Rev. Lett. 96, 147002 (2006). https://doi.org/10.1103/PhysRevLett.96.147002

24. Vercruyssen, N., Verhagen, T. G. A., Flokstra, M. G., Pekola, J. P. \& Klapwijk, T. M. Evanescent states and nonequilibrium in driven superconducting nanowires. Phys. Rev. B 85, 224503 (2012). https://doi.org/10.1103/PhysRevB.85.224503

25. Brinkman, A., Golubov, A. A., Rogalla, H., Wilhelm, F. K. \& Kupriyanov, M. Yu. Microscopic nonequilibrium theory of double-barrier Josephson junctions. Phys. Rev. B 68, 224513 (2003). https://doi.org/10.1103/PhysRevB.68.224513

26. Bezuglyi, E. V., Vasenko, A. S., Shumeiko, V. S. \& Wendin, G. Nonequilibrium effects in tunnel Josephson junctions. Phys. Rev. B 72, 014501 (2005). https://doi.org/10.1103/PhysRevB.72.014501

\section{Additional information}

The author declares no competing interests. 